\title{
A Study on Visual and Structural Characteristics of Landmarks and Experts' and Non-experts' Evaluations
}

\author{
Demet Yesiltepe $^{11}$ ', Ruth Conroy Dalton ${ }^{2}$, Ayse Ozbil Torun ${ }^{1}$, \\ Antoine Coutrot ${ }^{3}$, Michael Hornberger ${ }^{4}$, and Hugo Spiers ${ }^{5}$ \\ ${ }^{1}$ Department of Architecture, Northumbria University, \\ Newcastle upon Tyne NE1 8ST, UK \\ \{demet.yesiltepe, ayse.torun\}@northumbria.ac.uk \\ 2 Department of Architecture, University of Lancaster, Lancaster LA1 4YW, UK \\ r.daltonl@lancaster.ac.uk \\ ${ }^{3}$ Laboratoire des Sciences du Numérique de Nantes, \\ Nantes University, CNRS, Nantes, France \\ antoine.coutrot@ls2n. fr \\ ${ }^{4}$ Norwich Medical School, University of East Anglia, Norwich NR4 7TJ, UK \\ m.hornberger@uea.ac.uk \\ 5 Department of Experimental Psychology, University College London, \\ London WC1E 6BT, UK \\ h.spiers@ucl.ac.uk
}

\begin{abstract}
The aim of this study is to understand what makes a landmark more salient and to explore whether assessments of saliency vary between experts and non-experts. We hypothesize that non-experts' saliency judgments will agree with those of the experts. Secondly, we hypothesize that not only visual characteristics but also structural characteristics make landmarks salient and that the size and visibility of objects are important for them to be considered as salient. To test our hypotheses, an online navigation game, Sea Hero Quest (SHQ), was used and two levels of the game were selected as the case study. The characteristics of these levels were evaluated by non-experts and experts in the field. Our results suggest that both visual and structural characteristics of landmarks make them more salient. We also discovered that experts' saliency evaluations are mostly consistent with non-experts'.
\end{abstract}

Keywords: Landmarks $\cdot$ Saliency $\cdot$ Navigation $\cdot$ Wayfinding

\section{Introduction}

Landmarks, as components of environments, play an important role in wayfinding tasks. They can be used in wayfinding tasks to identify specific points [1], understand whether or not the followed path is correct [2, 3], organize spatial knowledge [4], change the position along a route [2], or learn a new route [5]. Therefore, they help people to find their way through different processes. However, it is still not completely clear what makes a landmark selected and preferred by more people for route 
instructions, orientation, or for any other wayfinding-related purpose. This study aims to better understand the characteristics of landmarks that make them salient. Moreover, we aim to understand whether landmark evaluations vary between experts working on wayfinding-related studies and lay people (non-experts). This has not been much studied.

\section{Background}

The characteristics of landmarks that cause them to be selected/preferred by more people were analyzed by past research which focused on the visual, structural and cognitive characteristics of objects [6]. Visual landmarks are distinguished by their physical characteristics such as color, size or shape. Cognitive landmarks are more personal; they frequently have a cultural or historical meaning. Hence, even if an object lacks visual attractiveness it can still be used by an observer to way-find. A structural landmark is selected because of its strategic location in an environment. The definition of saliency was further refined by Caduff and Timpf [7] who stated that methods should be suggested to measure saliency quantitatively. They introduced three terms of saliency: perceptual, cognitive and contextual. Taking a similar approach to Sorrow and Hirtle, they identified the physical characteristics of objects for describing perceptual salience. They extended the definition by describing three categories of perceptual salience: location-based (color, intensity, texture orientation), scene-context (topology and metric refinements) and object-based (size, shape and object orientation). Two components were identified for cognitive salience: the degree of recognition (indicating how well objects can be identified from others) and idiosyncratic relevance (the personal importance of objects for observers). For contextual saliency, researchers focused on two types of contexts: task-based context, which includes the types of tasks, and modality-based context, which includes the mode of transportation and the number of resources. By using these terms, further studies aimed to explain the most effective saliency criteria for wayfinding. Results showed that structural salience [8, 9], visibility (the ability to see a landmark from various points) [8, 10] and/or color [10] were potentially the most effective during a wayfinding task.

On the other hand, a limited number of studies focused on the combined impact of landmarks [2, 11]. Albrecht and Von Stülpnagel [11] aimed to explore the combined effect of visual and structural salience on wayfinding. They located visually salient objects both at structurally strategic locations and structurally less strategic locations. Researchers discovered that people tend to remember a turn correctly if a visually salient landmark is located in the turning direction. Similarly, Michon and Denis [2] asked twenty people to learn two routes by navigation and to generate route directions. Researchers observed that visual landmarks are better remembered when they are close to junctions. Thus, both studies lead to the hypothesis that visually salient landmarks are preferred and/or selected more frequently when they are located at structurally salient locations. Still, there is not a sufficient number of papers on the combined characteristics of landmarks. This study is therefore unique in considering the combined effect of two criteria. 
Moreover, the number of studies on experts' and non-experts' evaluations on landmarks is quite limited. Previous studies argued that experts' evaluations on environment can differ from those of non-experts' [12] since experts' evaluations involve complex considerations, such as aesthetics, creativity or functionality, while nonexperts' evaluation mostly focus on perception of the environment [13]. An interesting study was conducted by Cheng to analyze landmarks through experts' and non-experts' perceptions [14]. Two groups were used for this study: the expert group was defined as consisting of landscape architects who had lived and worked in the study area for over ten years; the non-expert group was defined as being local residents who lived in the study area for again more than ten years. Both groups answered questions about landmarks and the results of the study showed that singularity (a sharp visual contrast with the background) and spatial prominence (the location of landmarks- whether or not they are visible from many points) were influential in participants' landmark identification. In addition, the results of the research showed similarities and differences between two groups.

In this study, we aim to understand the characteristics of landmarks that make them salient as well as to explore the combined characteristics of landmarks. Moreover, by asking two different groups (experts and non-experts) their landmark evaluations, we aim to identify the similarities and differences between the evaluations by these two groups.

\section{Method}

An online game, Sea Hero Quest (SHQ), was selected as a case study [15]. The game was released in 2016 and more than 4.3 million people downloaded and played it. Seventy-five levels (and environments) were designed for the game. In wayfinding levels, which are used in this study, participants were first asked to view a map where they could see the start point of the wayfinding task, the environment that they would move in and the locations of the numbered buoys that they should find. Then they closed the map and started navigating a boat in a river/waterway environment and finding the buoys. Not only the environments but also the weather, map and landmark conditions varied in levels. For example, in certain levels the weather was clear (so that people could see their surroundings easily) while in others it was foggy (so that visibility was reduced and people could only see their immediate surroundings clearly); in some levels the water-course was wavy (and hence visibility changes constantly). The map condition was either clear (so the layout and the checkpoints could be seen clearly) or partially obscured (the layout couldn't be seen clearly, only the checkpoints could be viewed). The saliency of landmarks also varied between "none" (no landmarks), "hard" and "easy landmarks". Salient (easy) landmarks in the game are defined with visually salient objects [6] that are located at accessible points. Less salient (hard) objects referred to salient or less salient objects located at segregated points, as rated by experts in the field. 


\subsection{Selection of Stimuli}

We used two levels of SHQ, where: 1) the layout of levels are as similar as possible -we used space syntax axial [16] and segment based analysis as well as complexity measures to define similar layouts; 2) the conditions are the same, while 3) saliency of landmarks varies as salient and less salient (Fig. 1).

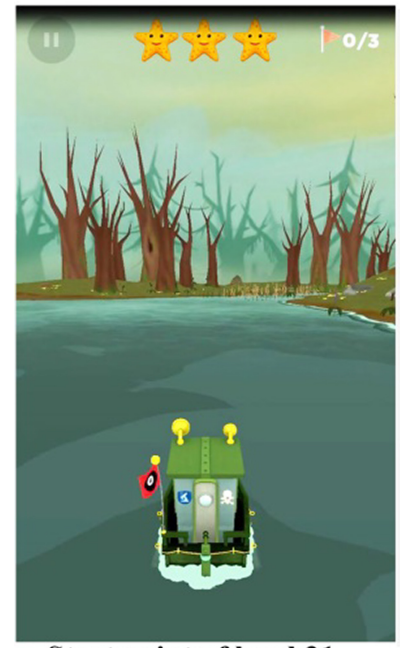

Start point of level 31

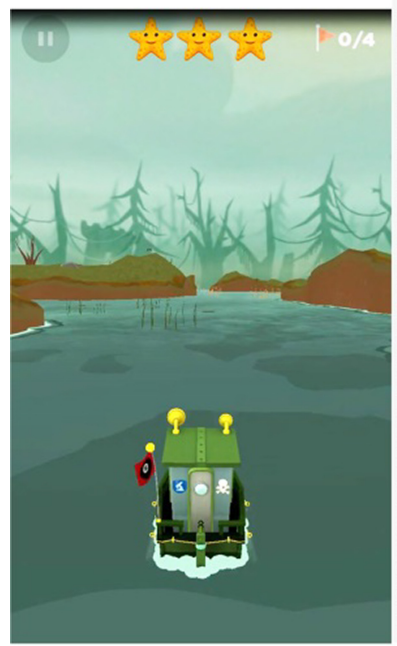

Start point of level 32

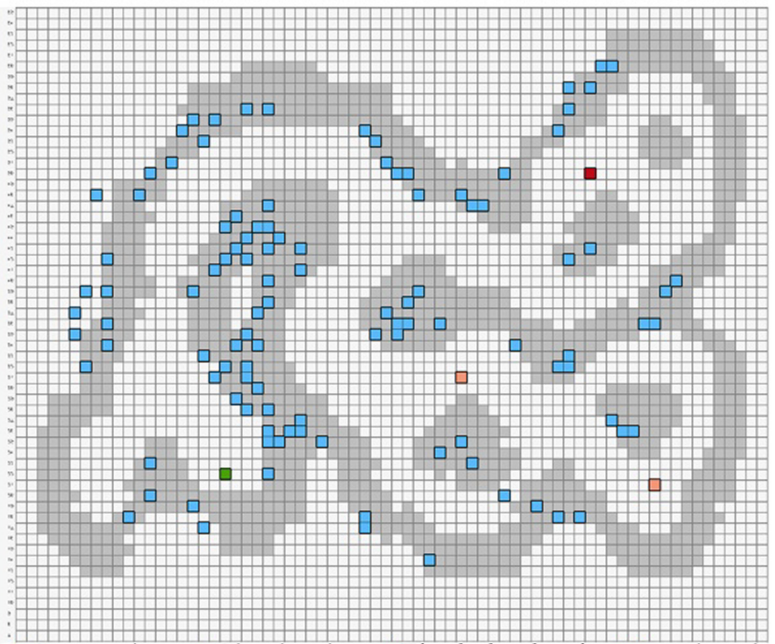

$=$ Start point $=$ Checkpoints $\square$ Final checkpoint $=$ Landmarks

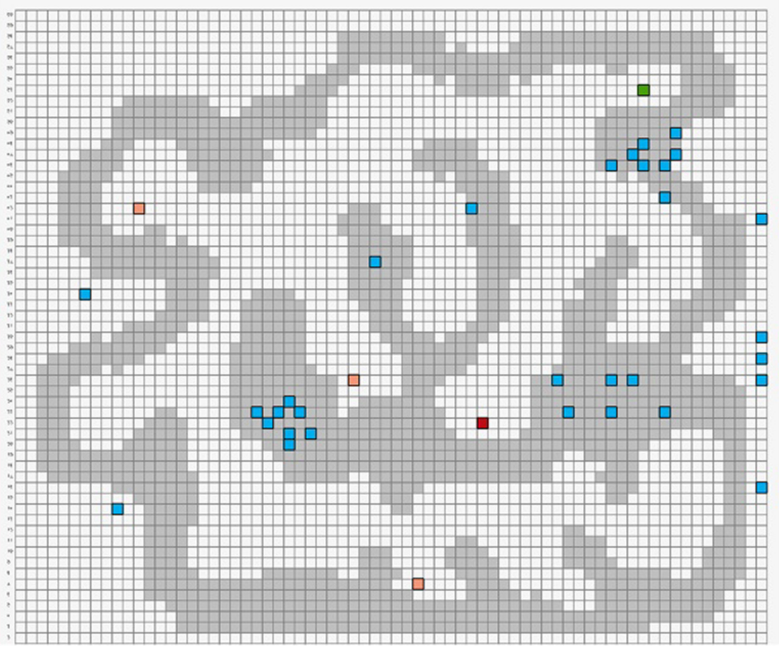

$=$ Start point $\equiv$ Checkpoints $₫$ Final checkpoint $₫$ Landmarks

Fig. 1. Layout of levels 31 and 32 and position of landmarks: screenshots were taken from the start points of level 31 (above) and 32 (below) and the start points, checkpoints, and final checkpoints were shown on the maps 
Space syntax measures included axial and segment based integration and choice (r: n, 3), axial based intelligibility, VGA (visual connectivity, visual integration, intelligibility), and connectivity (directional reach based on $10^{\circ}$ for 0 and 2 direction changes, metric reach for $10 \mathrm{~m}$ and $100 \mathrm{~m}$ ), whereas complexity measures included the number of decision points and destinations, total segment length, and shortest route. Clustering was conducted by using these measures to enable similar layouts to be selected [17]). Levels 31 and 32 are selected because they were in the same clustergroup of similar spatial properties and these levels included the same landmarks (there were only two additional landmarks in level 31) but which were located in different positions (different structural saliency). Level 31 had easy landmark condition and level 32 had hard landmark condition.

\subsection{Survey Design}

Once the levels were selected, a video was recorded for each level in which the boat moved and located all buoys in turn. Then the screenshots were taken from each video (from approximately same distance) for each landmark to show the participants. Two images were created for each landmark; in one, participants could see the image of a landmark as they saw it in the video; in the second one, a transparent image was used where participants could focus on the landmark that they were being asked to evaluate (Fig. 2). The consent form was approved by Northumbria University's Ethics Committee.

In the beginning of the survey, participants were asked to answer questions about data protection and participation as well as demographics. They were informed that they could leave the survey at any time and that their data would be anonymized. Then they were asked to watch the videos respectively and pay attention to the environment through which the boat moved. They were asked to watch the videos before they moved to the next section of the questionnaire. When they finished watching the

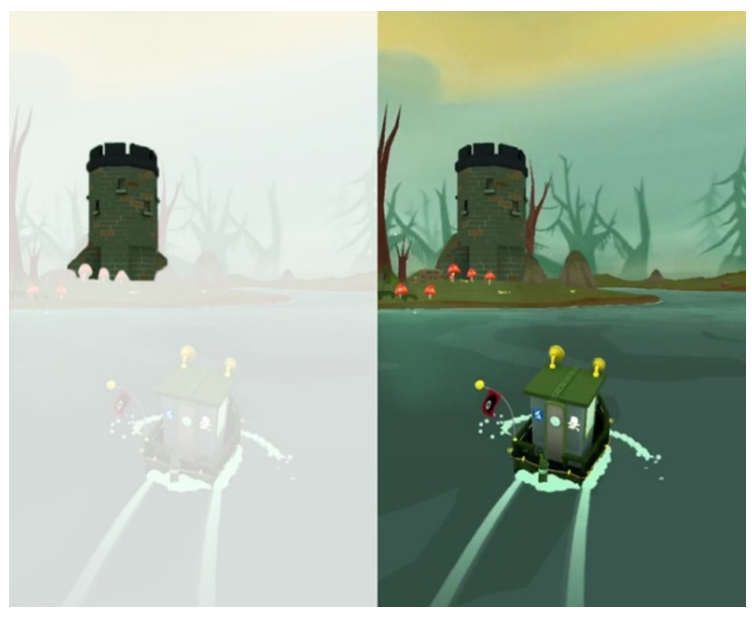

Fig. 2. Images of landmarks that are shown to participants (on left, background is transparent so that the landmark can be clearly seen and on right, the scene is directly taken from the video). 
videos, they were shown the images of landmarks in a randomized order and were asked to categorize landmarks using a 5-point Likert scale from 1 (unnoticeable) to 5 (highly noticeable). For each landmark, we already had ratings (as 1: salient and 0: less salient landmarks) provided by the experts, since they evaluated landmarks during the design phase of the game.

\subsection{Participants}

The web-based survey (Google survey) was prepared and participants were recruited online via a range of social media channels (from March $22^{\text {nd }}$ to April $5^{\text {th }} 2019$ ). These comprise the non-expert participants of the study. 251 people aged between 18 and 70 attended the survey. This number included 165 female, 84 male participants ( 2 participants preferred not to mention).

On the other hand, 4 experts took part in the study. Experts in navigation studies, who worked on wayfinding or navigation fields for over ten years, were selected from different disciplines (architecture, psychology, cognitive science) and different universities. They agreed on taking part in the study and evaluate the landmarks. While evaluating the landmarks, experts viewed them on a white background and evaluated them out of context (i.e. rather than viewing them in their final game-environment context).

\section{Results}

First, Cronbach's alpha was calculated in SPSS to test the reliability coefficient for internal consistency of the survey results. Cronbach's alpha was .74 when all landmarks and all participants' evaluations were used, indicating that the questionnaire has good internal consistency. Hence all results are included in the study. Reliability test was not conducted for the evaluation of the experts since they agreed on the saliency of landmarks. Results of the survey study showed that size and color of objects are important for them to be chosen as salient objects (see Fig. 3 and Fig. 4). Castle, grass and trees were selected as salient objects in both levels. This was followed by arch and toadstool in level 31 and by toadstool in level 32. Small stone and plant were rated as highly noticeable by a limited number of people. 


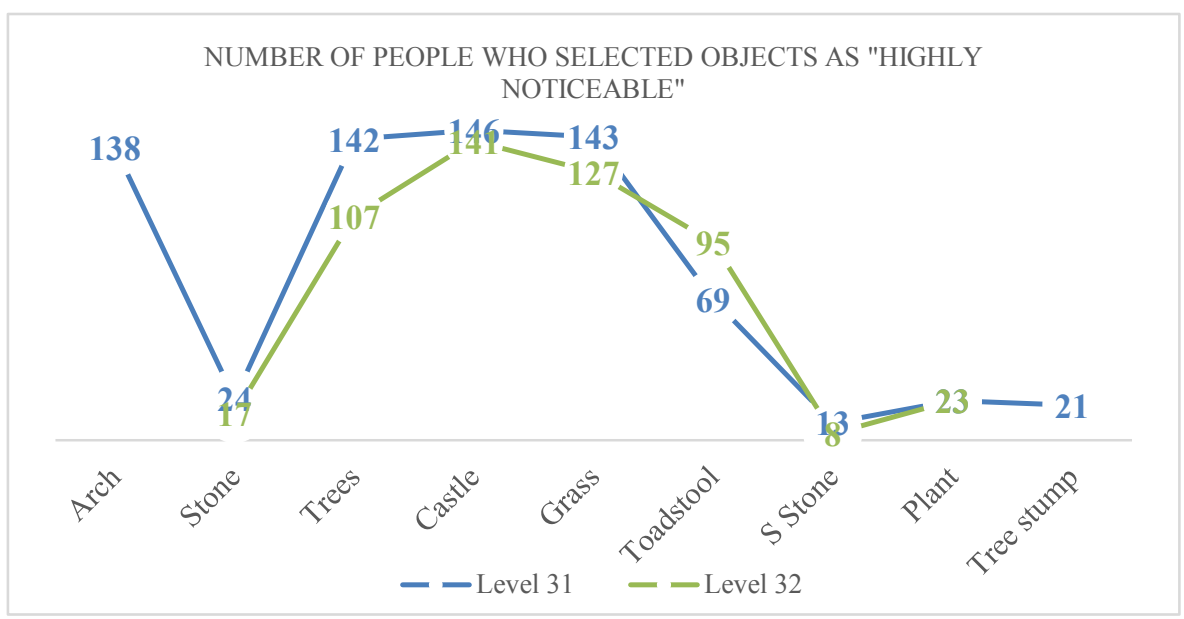

Fig. 3. "Highly noticeable" objects for levels 31 and 32 for non-experts' (there was no tree stump or arch in level 32)

When the landmarks are viewed within their context, it can be seen that highly rated objects tend to stand out from their surroundings particularly due to their size and color. The toadstool and trees contrast with the background while the castle, tree and arch differentiate from their surroundings particularly due their size. The least rated objects, on the other hand, are smaller objects with a similar color with the background. Hence, we can assume that it was harder for participants to notice these objects.

The ratings show that the number of objects that are rated as "highly noticeable" is higher in level 31 compared to level 32 (except for toadstool). When the videos are played again, it was seen that the boat moved quite close to the toadstools in level 32 (Fig. 5). Hence, participants could have more of an opportunity to see this landmark more closely, which may account for this unexpected finding. In addition, in level 31 the toadstools were viewed along with various other landmarks, while in level 32 they were seen on their own. This can support the findings of previous studies $[18,19]$, where researchers mentioned that the existence of salient landmarks can make other landmarks less salient. For the other landmarks, however, we can claim the impact of structural saliency on participants' rates. 


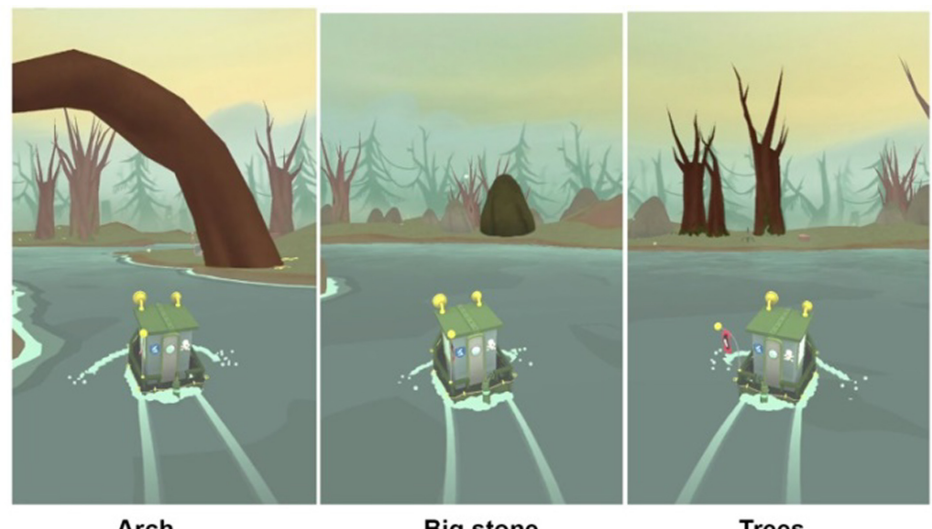

Arch

Big stone

Trees

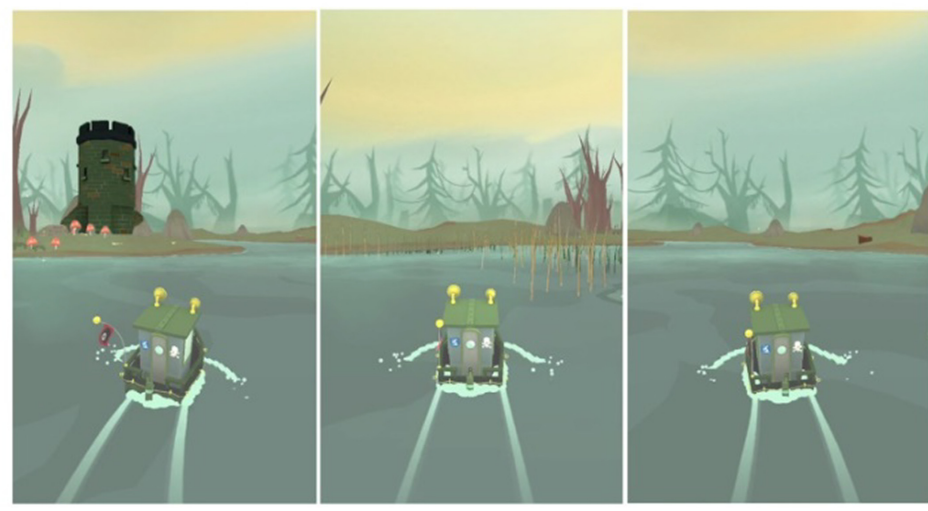

Castle

Grass

Tree stump

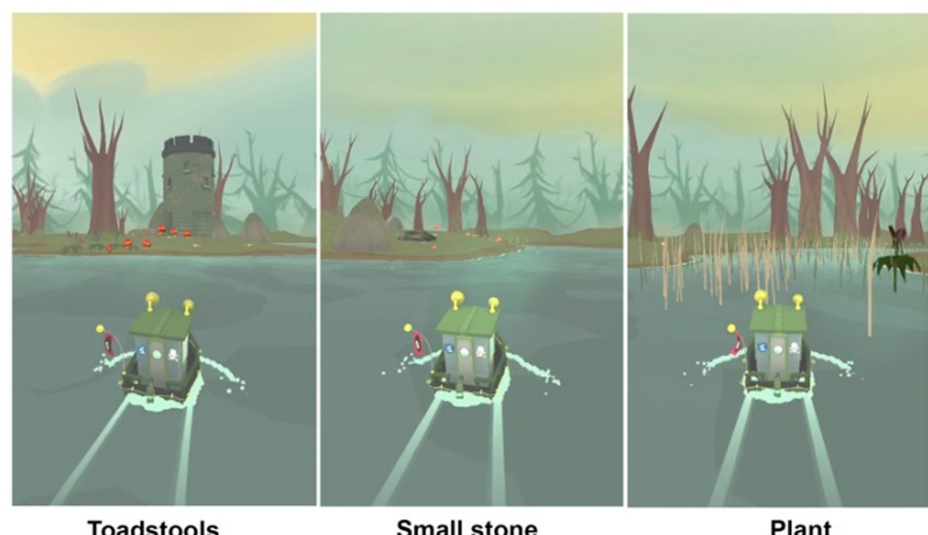

Fig. 4. All landmarks that are shown to participants; images are taken from level 31 (all shown within context and with a transparent background) 


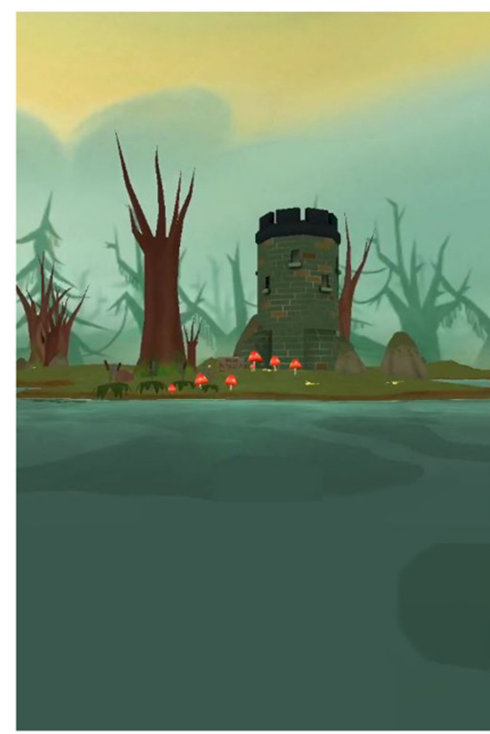

Level 31

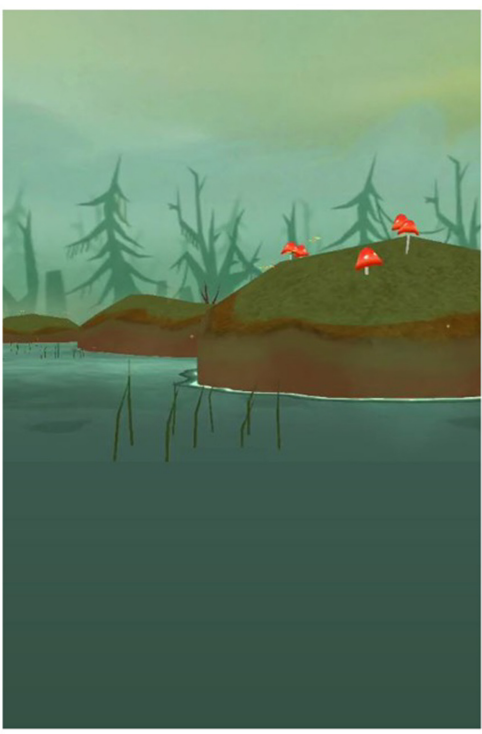

Level 32

Fig. 5. The image used in level 31 and 32 to evaluate the toadstool

In addition, we compared the experts' results with those of non-experts'. Experts' saliency evaluation included two categories: salient objects (1) and less salient objects (0). Hence, non-experts' evaluations were also categorized as salient and less salient objects. Figure 6 shows the results of two groups together.

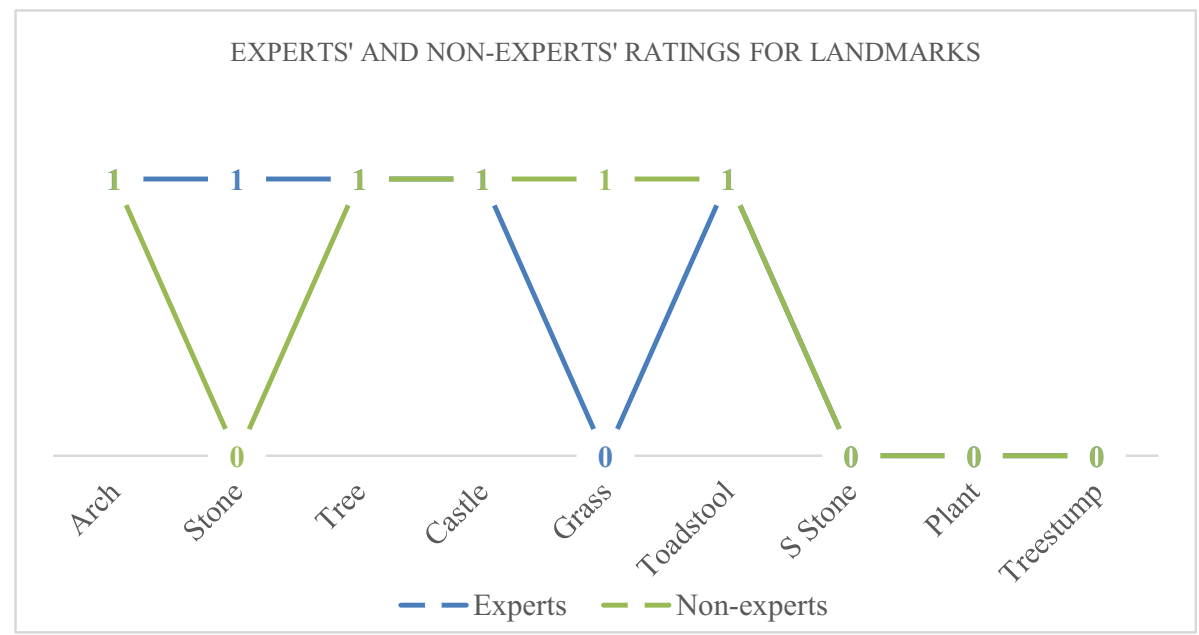

Fig. 6. Results of experts' and non-experts' evaluations. "1" represents salient landmarks and " 0 " represents less salient landmarks. 
Results suggest that the ratings of both groups (experts and non-experts) are the same for all landmarks, except for stone and grass. While the stone was selected as a salient and a less salient object by experts and non-experts respectively, the opposite was true for grass (i.e. grass was selected as a salient object by non-experts while experts rated it as less salient).

\section{Discussion}

This study aimed to better understand the properties of landmarks, which make them salient. In particular, we focused on the visual and structural characteristics of landmarks. An online game, Sea Hero Quest was used for this purpose and objects, which varied in terms of their shape, size and color, and which were located at different points in two levels, were used as landmarks. Two levels of the game were selected based on their spatial values (levels with similar values were chosen) and their conditions (all conditions were the same except landmark condition). The landmark conditions of levels 31 and 32 were defined as "easy" and "hard" respectively by a group of experts who designed the layouts of levels. Therefore, we used these two levels and asked participants to evaluate saliency of landmarks located within the different environment of these levels.

First of all, results of this study pointed to different objects being rated as salient landmarks: the castle, trees, grass and arch were defined as the highly noticeable objects by a high number of participants. Trees, castle and the arch differentiated from their surroundings with their height and color. Therefore, our findings indicate that color (and its contrast with the background) and size are significant visual characteristics of landmarks. These results were in agreement with the findings of past research [10]. Moreover, in line with the experts' ratings, non-experts identified that objects in level 32 were less salient. Only the toadstools, which were rated as a salient object by participants, were not supported by this finding. When we viewed the videos again, we observed that unlike the other objects, in level 32, the toadstools were closer to the screen (so they could be seen more easily). This result is very important because while the landmarks were consistent between levels, their location was altered. In other terms, their visual saliency was the same while their structural saliency differed. This implies that changing the structural saliency can affect people's perception on visual saliency. This finding replicated the findings of the previous research $[2,11]$.

When we focused on structural saliency, on the other hand, it was observed that non-experts' results were in line with those of experts'. The objects in level 32 (the level with low accessibility, according to the experts) were rated as highly significant by a lower number of people, compared to level 31. Only one group of objects, toadstools, was evaluated differently out of the nine. When we focused on the underlying reason, we recognized that the position of toadstools changed significantly in two levels: in level 31 toadstools were further from the screen and they were located together with some other salient landmarks (castle, trees). We suggest that changing the structural saliency could have led to higher ratings. In addition, when we compared the two levels, in level 32 the number of landmarks decreased (no arch and tree stump was used) and the location of landmarks also changed (Fig. 7). As both of these can be 
important factors, when the two videos were viewed again, it was better seen that the location of landmarks were significantly different in two levels. In one, level 31, objects were on route, visible from many angles and close to the observers (so that they could be seen from shorter distances, and also over multiple times) while in the other level, level 32, trees, castle and stone (some of the salient landmarks of level 31) were further from the route with a limited visibility. Hence, it can be argued that the location of an object is also effective in identifying the object as a salient one, as mentioned in the literature [9].

In addition, we discovered that the saliency judgements by the experts were in line with the non-experts' survey results, as we expected. In the literature, past studies found similarities and differences between two groups [14]. In this study, we found that the experts' results could explain the survey results of non-experts for many landmarks. However, there were differences between the ratings of two landmarks. The reason for the differentiation can be explained with the "context" based limitations [7]. While nonexperts were able to view the landmarks in the game environment, experts viewed the landmarks on a white background out of their context (because these judgements had been made during the game-development process and not after its completion). Moreover, the experts viewed only images of the landmarks, while non-experts viewed a video in which landmarks were located throughout the video. We believe this difference between the modes of viewing landmarks may also have caused differences in their evaluations. Hence, this can be accepted as one of the limitations of the current study.

The sample size of the landmarks was another limitation of this study (9 landmarks in level 31 and 7 in level 32). Further studies need to be conducted using a higher number of landmarks. Furthermore, we compared the results of 251 non-experts with 4 experts. More research, including a higher number of experts, could be undertaken to explore the differences in the judgements of the two groups.

Despite these limitations, this study contributes to the literature by highlighting the role of the visual and structural saliency of landmarks within two virtual environments in the evaluations by experts versus non-experts. Since the number of studies about the combined impact of landmarks is limited, we believe this study is an important contribution to the literature. 


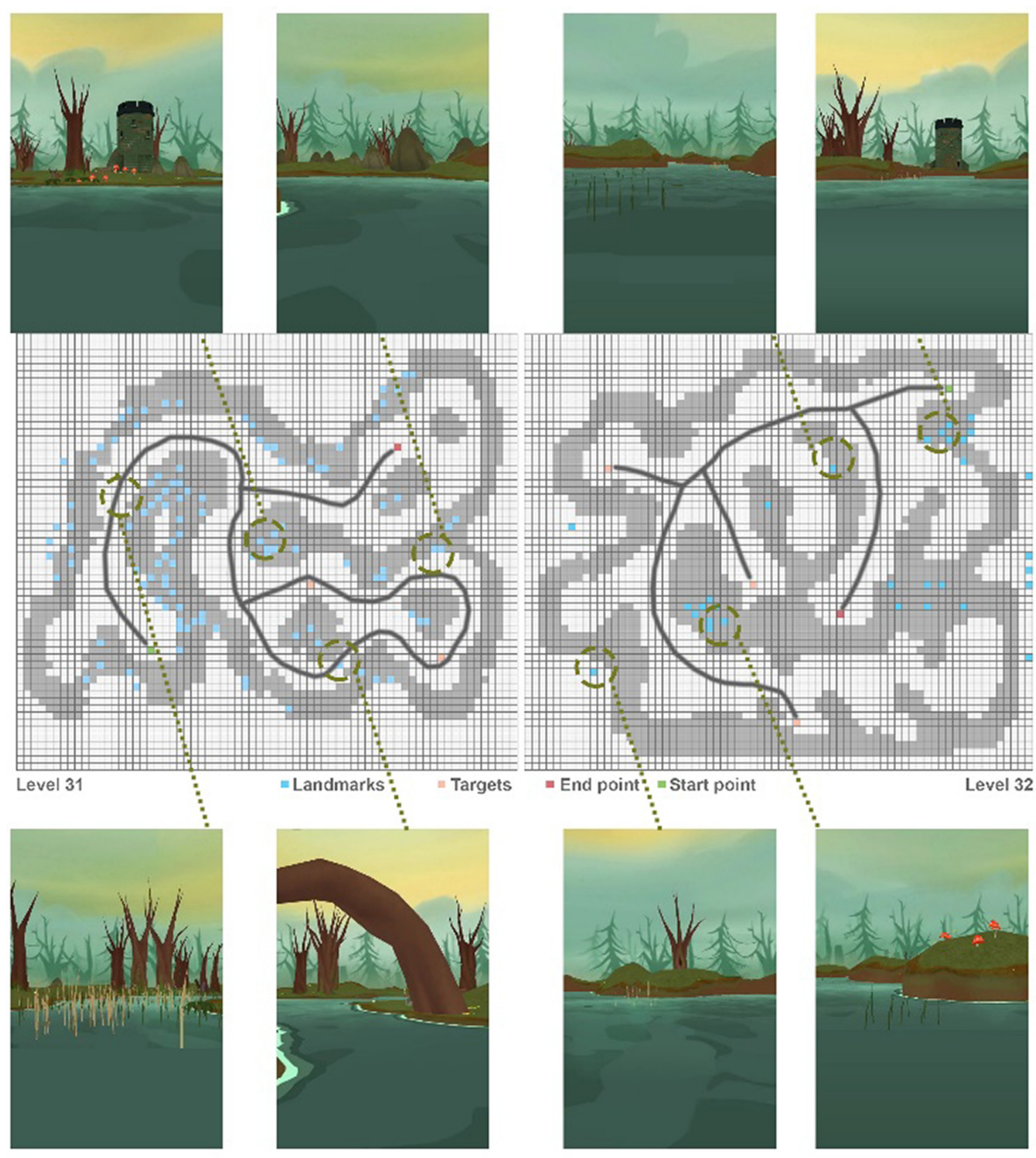

Fig. 7. Layout of two levels and the location of landmarks

Acknowledgment. The authors thank Saskia Kuliga for her suggestions about the survey.

\section{References}

1. Downs, R.M., Stea, D.: Cognitive maps and spatial behavior: process and products. In: The Map Reader: Theories of Mapping Practice and Cartographic Representation. pp. 8-26. Wiley (2011). https://doi.org/10.1002/9780470979587.ch41

2. Michon, P.-E., Denis, M.: When and why are visual landmarks used in giving directions? In: Montello, D.R. (ed.) Spatial Information Theory, pp. 292-305. Springer, Heidelberg (2001) 
3. Philbeck, J.W., O'Leary, S.: Remembered landmarks enhance the precision of path integration. Psicologica 26, 7-24 (2005)

4. Couclelis, H., Golledge, R.G., Gale, N., Tobler, W.: Exploring the anchor-point hypothesis of spatial cognition. J. Environ. Psychol. 7, 99-122 (1987). https://doi.org/10.1016/S02724944(87)80020-8

5. Tlauka, M., Wilson, P.N.: The effect of landmarks on route-learning in a computer-simulated environment. J. Environ. Psychol. 14, 305-313 (1994)

6. Sorrows, M.E., Hirtle, S.C.: The nature of landmarks for real and electronic spaces. In: International Conference on Spatial Information Theory. pp. 37-50. Springer, Heidelberg (1999). https://doi.org/10.1007/3-540-48384-5

7. Caduff, D., Timpf, S.: On the assessment of landmark salience for human navigation. Cogn. Process. 9, 249-267 (2008). https://doi.org/10.1007/s10339-007-0199-2

8. Peters, D., Wu, Y., Winter, S.: Testing landmark identification theories in virtual environments. In: Hölscher, C., Shipley, T.F., Olivetti Belardinelli, M., Bateman, J.A., Newcombe, N.S. (eds.) Spatial Cognition VII, pp. 54-69. Springer, Heidelberg (2010)

9. Stankiewicz, B.J., Kalia, A.A.: Acquistion of structural versus object landmark knowledge. J. Exp. Psychol. Hum. Percept. Perform. 33, 378-390 (2007)

10. Winter, S., Raubal, M., Nothegger, C.: Focalizing measures of salience for wayfinding. In: Map-based Mobile Services: Theories, Methods and Implementations. pp. 125-139. Springer, Heidelberg (2005). https://doi.org/10.1007/3-540-26982-7_9

11. Albrecht, R., von Stuelpnagel, R.: Memory for salient landmarks: empirical findings and a cognitive model. In: Creem-Regehr, S., Schöning, J., Klippel, A. (eds.) Spatial Cognition 2018. LNCS (LNAI), vol. 11034, pp. 311-325. Springer, Cham (2018). https://doi.org/10. 1007/978-3-319-96385-3_21

12. Hölscher, C., Dalton, R.C.: Comprehension of layout complexity: Effects of architectural expertise and mode of presentation. In: Gero, J.S., Goel, A.K. (eds.) Design Computing and Cognition 2008, pp. 159-178. Springer, Dordrecht (2008)

13. Tenbrink, T., Hoelscher, C., Tsigaridi, D., Dalton, R.: Cognition and communication in architectural design. In: Montello, D., Grossner, K., Janelle, D. (eds.) Space in Mind: Concepts for Spatial Learning and Education, pp. 263-280. The MIT Press, United States (2014). https://doi.org/10.7551/mitpress/9811.003.0014

14. Cheng, S.-Y.: Suburban landmarks in north arlington: perceptions of experts and non-experts (2009)

15. Coutrot, A., et al.: Global determinants of navigation ability. Curr. Biol. 28, 2861-2866.e4 (2018). https://doi.org/10.1016/J.CUB.2018.06.009

16. Hillier, B., Hanson, J.: The Social Logic of Space. Cambridge University Press, Cambridge (1984)

17. Yesiltepe, D., et al.: Usage of landmarks in virtual environments for wayfinding: research on the influence of global landmarks. In: 12th International Space Syntax Symposium, pp. 2201-220-10, Beijing (2019)

18. Raubal, M., Winter, S.: Enriching wayfinding instructions with local landmarks. In: Egenhofer, M.J., Mark, D.M. (eds.) GIScience 2002. LNCS, vol. 2478, pp. 243-259. Springer, Heidelberg (2002). https://doi.org/10.1007/3-540-45799-2_17

19. Sadeghian, P., Kantardzic, M.: The new generation of automatic landmark detection systems: challenges and guidelines. Spat. Cogn. Comput. 8, 252-287 (2008). https://doi.org/ $10.1080 / 13875860802039257$ 